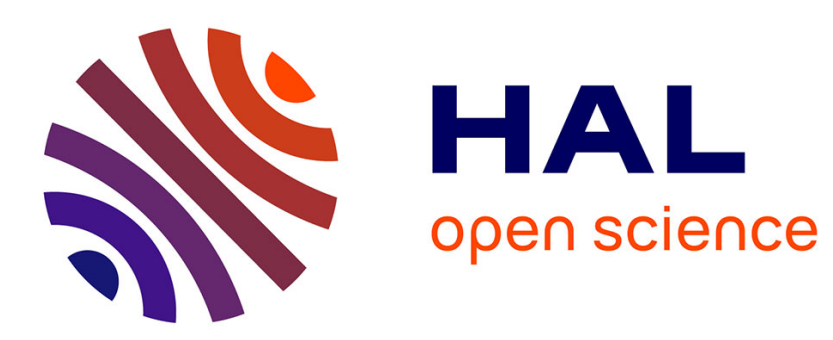

\title{
Improving the Performance of Space Shift Keying (SSK) Modulation via Opportunistic Power Allocation
}

\author{
Marco Di Renzo, Harald Haas
}

\section{To cite this version:}

Marco Di Renzo, Harald Haas. Improving the Performance of Space Shift Keying (SSK) Modulation via Opportunistic Power Allocation. IEEE Communications Letters, 2010, 11 (6), pp. 500-502. hal00547009

\section{HAL Id: hal-00547009 \\ https://hal.science/hal-00547009}

Submitted on 15 Dec 2010

HAL is a multi-disciplinary open access archive for the deposit and dissemination of scientific research documents, whether they are published or not. The documents may come from teaching and research institutions in France or abroad, or from public or private research centers.
L'archive ouverte pluridisciplinaire HAL, est destinée au dépôt et à la diffusion de documents scientifiques de niveau recherche, publiés ou non, émanant des établissements d'enseignement et de recherche français ou étrangers, des laboratoires publics ou privés. 


\title{
Improving the Performance of Space Shift Keying (SSK) Modulation via Opportunistic Power Allocation
}

\author{
Marco Di Renzo, Member, IEEE, and Harald Haas, Member, IEEE
}

\begin{abstract}
In this Letter, we show that the performance of Space Shift Keying (SSK) modulation can be improved via opportunistic power allocation methods. For analytical tractability, we focus on a $2 \times 1$ Multiple-Input-Multiple-Output (MIMO) system setup over correlated Rayleigh fading channels. A closedform solution of the optimal power allocation problem is derived, and it is shown that the transmit-power of each transmitantenna should be chosen as a function of the power imbalance ratio and correlation coefficient of the transmit-receive wireless links. Numerical results are shown to substantiate the analytical derivation and the claimed performance improvement.
\end{abstract}

Index Terms-Space Shift Keying (SSK) modulation, optimization, fading channels, opportunistic power allocation.

\section{INTRODUCTION}

$\mathbf{S}$ PACE MODULATION is a recently proposed wireless transmission concept for low-complexity implementations of Multiple-Input-Multiple-Output (MIMO) wireless systems [1]-[5], which combines digital modulation, coding, and multiple-antennas transmission in a unique fashion, and exploits the location-specific property of the wireless channel for communication [1]. Recent results have shown that it can yield better performance, with a reduced computational complexity, than other MIMO schemes [3]-[5].

Among the various solutions available in the literature to date (see, e.g., [6] for a survey), the schemes introduced in [3] and [5] offer two simple alternatives to circumvent the three main technological issues for a low-complexity and energy-efficient implementation of MIMO systems, i.e., InterChannel Interference (ICI), Inter-Antenna Synchronization (IAS), and multiple Radio Frequency (RF) chains at the transmitter [3], [5]. This is achieved by adopting a simple but effective coding mechanism that establishes a one-to-one mapping between blocks of information bits to be transmitted and the spatial positions of the transmit-antennas (the socalled "spatial constellation diagram"), which are used as an additional dimension for conveying information [3], [5]. More specifically, ICI, IAS and multiple RF chains at the transmitter are avoided by enabling just a single transmit-antenna to radiate power at any time-instant. The methods introduced in [3] and [5] are called Spatial Modulation (SM) and Space Shift Keying (SSK) modulation, respectively. Broadly speaking, SSK modulation [5] is a special case of SM [3], which can trade-off receiver complexity for data rate.

The performance studies conducted in [3], [5] implicitly assume that each transmit-antenna, when switched on for data

Manuscript received January 29, 2010. The associate editor coordinating the review of this letter and approving it for publication was R. Nabar.

M. Di Renzo is with L2S, UMR 8506 CNRS - SUPELEC - Univ ParisSud, 3 rue Joliot-Curie, 91192 Gif-sur-Yvette CEDEX, France (e-mail: marco.direnzo@1ss.supelec.fr).

H. Haas is with the Institute for Digital Communications (IDCOM), The University of Edinburgh, Edinburgh, UK (e-mail: h.haas@ed.ac.uk).

Digital Object Identifier 10.1109/LCOMM.2010.06.100163 transmission, radiates the same power. However, in [7] we have recently shown that the performance of SSK modulation depends on the difference between the complex channel gains of pairs of transmit-receive wireless links: the more different from each other they are, the better the performance is. In this regard, opportunistic power allocation methods could be exploited for an easier differentiation of the wireless links, by emphasizing the "propagation modes" along any transmit-receive wireless links according to the actual fading conditions. Of course, the price to be paid to enable this adaptation capability is the need of some a priori channel state information at the transmitter. However, we prove that only average channel state information is required to exploit the proposed method.

To keep the analytical complexity at a moderate level, in this Letter we restrict the analysis to a simple $2 \times 1$ MIMO scheme over correlated Rayleigh fading channels, which adopts the SSK modulation principle in [5]. With these assumptions, the specific contribution of this Letter is as follows: i) the optimal power allocation problem that minimizes the Average Bit Error Probability (ABEP) is solved in closed-form; ii) it is shown that SSK modulation with optimal power allocation resembles an On-Off Keying (OOK) modulation scheme, which, for equiprobable binary symbols, foresees to switch the first and second transmit-antennas on and off, respectively, if a binary " 0 " needs to be transmitted, and to switch both transmitantennas off if a binary " 1 " needs to be transmitted. Owing to this analogy, the new SSK modulation scheme is called OnOff SSK (OOSSK) modulation; iii) it is analytically proved that, for the same average transmit-power, the lowest ABEP is obtained by switching on the transmit-antenna corresponding to the transmit-receive wireless link having the best average Signal-to-Noise-Ratio (SNR); and iv) the performance gain (or energy saving) of OOSSK modulation with respect to SSK modulation is computed in closed-form and shown to depend on the power imbalance ratio and the spatial correlation coefficient of the wireless links. Finally, Monte Carlo simulations are shown to substantiate the analytical derivation.

\section{Problem Statement}

Let us consider a $N_{t} \times N_{r}$ MIMO system, where $N_{t}=2$ and $N_{r}=1$ are the transmit- and receive-antennas, respectively. SSK modulation works as follows [5]: i) the transmitter encodes blocks of $\log _{2}\left(N_{t}\right)$ data bits into the index of a single transmit-antenna, which is switched on for data transmission while all other antennas are kept silent, and ii) the receiver solves a $N_{t}$-hypothesis detection problem to estimate the transmit-antenna that is not idle, which results in the estimation of the sequence of bits emitted by the encoder. 


\section{A. Notation}

The notation adopted in this Letter is as follows: i) $E_{1}$ and $E_{2}$ denote the energies transmitted by the first and the second antenna when switched on for transmission, e.g., the first and second transmit-antenna is switched on if a binary " 0 " and " 1 " need to be transmitted, respectively [5]; ii) $\sigma_{1}^{2}$ and $\sigma_{2}^{2}$ are the parameters of the Rayleigh fading along the transmit-receive wireless link from the first and second transmit-antenna to the single receive-antenna, respectively; iii) $0 \leq \rho \leq 1$ is the spatial correlation coefficient between the two available transmit-receive wireless links (see [6] for definition); and iv) $N_{0}$ denotes the power spectral density of the Additive White Gaussian Noise (AWGN) at the receiver input.

\section{B. $A B E P$}

In [6], we have recently computed the ABEP of SSK modulation over correlated Rayleigh fading channels by assuming that each antenna, when switched on for data transmission, radiates the same power ${ }^{1}$, i.e., $E_{1}=E_{2}$. The framework in [6] can be readily generalized to the scenario where the transmitantennas can radiate a different power, as follows:

$$
\operatorname{ABEP}\left(E_{1}, E_{2}\right)=\frac{1}{2}-\frac{1}{2} \sqrt{\operatorname{SNR}\left(E_{1}, E_{2}\right)}
$$

where we have defined $\bar{\sigma}^{2}=E_{1} \sigma_{1}^{2}+E_{2} \sigma_{2}^{2}-2 \rho \sqrt{E_{1}} \sqrt{E_{2}} \sigma_{1} \sigma_{2}$, $\bar{\gamma}=1 /\left(4 N_{0}\right)$, and $\operatorname{SNR}\left(E_{1}, E_{2}\right)=\bar{\sigma}^{2} \bar{\gamma} /\left(1+\bar{\sigma}^{2} \bar{\gamma}\right)$ is the equivalent end-to-end SNR of the MIMO system.

Unlike current results available in the literature [3], [5], in this Letter we aim at computing the optimal allocation of $\left(E_{1}, E_{2}\right)$ over the two transmit-antennas, with the goal to minimize the ABEP in (1) for a given power budget at the transmitter. In formulas, the problem can be formulated as:

$$
\left\{\begin{array}{l}
\left(E_{1}^{*}, E_{2}^{*}\right)=\underset{\left(E_{1}, E_{2}\right)}{\arg \min }\left\{\operatorname{ABEP}\left(E_{1}, E_{2}\right)\right\} \\
\text { subject to : } \frac{E_{1}}{2}+\frac{E_{2}}{2}=E_{\mathrm{av}}
\end{array}\right.
$$

where $\left(E_{1}^{*}, E_{2}^{*}\right)$ denotes the optimal value of $\left(E_{1}, E_{2}\right)$ that solves the constrained optimization problem in (2), and $E_{\mathrm{av}}$ is the average energy consumed per transmission. Also, let us note that the power constraint in (2) corresponds to keeping constant the average power consumed per transmission for equiprobable binary symbols. All results available for SSK modulation to date simply assume $E_{1}=E_{2}=E_{\text {av }}$ [6].

Since the ABEP in (1) is a decreasing function of the equivalent end-to-end $\operatorname{SNR}, \operatorname{SNR}(\cdot, \cdot)$, then the optimization problem in (2) can be simplified as follows:

$$
\left\{\begin{aligned}
\left(E_{1}^{*}, E_{2}^{*}\right)= & \underset{\left(E_{1}, E_{2}\right)}{\arg \max }\left\{\operatorname{SNR}\left(E_{1}, E_{2}\right)\right\} \\
\text { subject to }: & E_{1}+E_{2}=2 E_{\mathrm{av}}
\end{aligned}\right.
$$

\section{Optimal Power Allocation}

The constrained optimization problem in (3) can be solved by using the Lagrange multiplier maximization method [8], which reduces to the computation of the maximum of the Lagrangian function, $\Lambda(\cdot, \cdot, \cdot)$, as follows:

$$
\Lambda\left(E_{1}, E_{2}, \lambda\right)=\operatorname{SNR}\left(E_{1}, E_{2}\right)+\lambda\left(E_{1}+E_{2}-2 E_{\text {av }}\right)
$$

\footnotetext{
${ }^{1}$ Since the signaling interval for all symbols is the same, the terms energy and power can be used interchangeably in this Letter.
}

where $\lambda$ is the Lagrange multiplier.

The stationary points of (4), which include the maximum of (3) that solves the original optimization problem in (2), can be computed by solving the system of equations in what follows:

$$
\left\{\begin{array}{l}
\partial \Lambda\left(E_{1}^{s}, E_{2}^{s}, \lambda^{s}\right) / \partial E_{1}=0 \\
\partial \Lambda\left(E_{1}^{s}, E_{2}^{s}, \lambda^{s}\right) / \partial E_{2}=0 \\
\partial \Lambda\left(E_{1}^{s}, E_{2}^{s}, \lambda^{s}\right) / \partial \lambda=0
\end{array}\right.
$$

where $\lambda^{s}$ is the Lagrange multiplier associated to the stationary point $\left(E_{1}^{s}, E_{2}^{s}\right)$. Among the set of stationary points $\left(E_{1}^{s}, E_{2}^{s}, \lambda^{s}\right)$ solving (5), the optimal power allocation $\left(E_{1}^{*}, E_{2}^{*}\right)$ in (3) can be obtained either by using the second derivative criterion or by direct inspection of the objective function $\operatorname{SNR}(\cdot, \cdot)$ in (3) [8]. For analytical simplicity, in this Letter we adopt the latter method.

\section{A. Stationary Points}

Three cases need to be distinguished to compute all stationary points of (5): 1) $E_{1} \neq 0$ and $E_{2} \neq 0$;2) $E_{1}=0$ and $E_{2}=2 E_{\mathrm{av}}$; and 3) $E_{1}=2 E_{\mathrm{av}}$ and $E_{2}=0$. Note that the special cases 2) and 3) need to be distinguished from case 1) because the partial derivatives in (5) cannot be computed if either $E_{1}$ or $E_{2}$ are equal to zero.

1) $E_{1} \neq 0$ and $E_{2} \neq 0$ : In this case, it can be shown, after a few algebraic manipulations, that (5) admits the two stationary points $S_{1}$ and $S_{2}$ as follows:

$$
\left\{\begin{array}{l}
E_{1}^{s_{1}, s_{2}}=E_{\mathrm{av}}\left[1 \pm \sqrt{\frac{\left(\sigma_{1}^{2}-\sigma_{2}^{2}\right)^{2}}{\left(\sigma_{1}^{2}-\sigma_{2}^{2}\right)^{2}+4 \rho^{2} \sigma_{1}^{2} \sigma_{2}^{2}}}\right] \\
E_{2}^{s_{1}, s_{2}}=E_{\mathrm{av}}\left[1 \mp \sqrt{\frac{\left(\sigma_{1}^{2}-\sigma_{2}^{2}\right)^{2}}{\left(\sigma_{1}^{2}-\sigma_{2}^{2}\right)^{2}+4 \rho^{2} \sigma_{1}^{2} \sigma_{2}^{2}}}\right]
\end{array}\right.
$$

2) $E_{1}=0$ and $E_{2}=2 E_{\mathrm{av}}$ : In this case, $E_{1}^{s_{3}}=0, E_{2}^{s_{3}}=$ $2 E_{\text {av }}$, and the end-to-end SNR in (3) reduces to:

$$
\operatorname{SNR}\left(E_{1}^{s_{3}}, E_{2}^{s_{3}}\right)=2 \bar{\gamma} \sigma_{2}^{2} E_{\mathrm{av}} /\left(1+2 \bar{\gamma} \sigma_{2}^{2} E_{\mathrm{av}}\right)
$$

3) $E_{1}=2 E_{\mathrm{av}}$ and $E_{2}=0$ : In this case, $E_{1}^{s_{4}}=2 E_{\mathrm{av}}$, $E_{2}^{s_{4}}=0$, and the end-to-end SNR in (3) reduces to:

$$
\operatorname{SNR}\left(E_{1}^{s_{4}}, E_{2}^{s_{4}}\right)=2 \bar{\gamma} \sigma_{1}^{2} E_{\mathrm{av}} /\left(1+2 \bar{\gamma} \sigma_{1}^{2} E_{\mathrm{av}}\right)
$$

\section{B. Computation of $\left(E_{1}^{*}, E_{2}^{*}\right)$}

Via direct inspection of $\left\{\operatorname{SNR}\left(E_{1}^{s_{i}}, E_{2}^{s_{i}}\right)\right\}_{i=1}^{4}$, it can be readily proved that the optimal solution of (3) is as follows.

1) $\sigma_{1}^{2}>\sigma_{2}^{2}$ : In this case, it can be verified that $\operatorname{SNR}\left(E_{1}^{s_{4}}, E_{2}^{s_{4}}\right)>\operatorname{SNR}\left(E_{1}^{s_{3}}, E_{2}^{s_{3}}\right)$ and $\operatorname{SNR}\left(E_{1}^{s_{4}}, E_{2}^{s_{4}}\right)>$ $\operatorname{SNR}\left(E_{1}^{s_{1}}, E_{2}^{s_{1}}\right)>\operatorname{SNR}\left(E_{1}^{s_{2}}, E_{2}^{s_{2}}\right)$. So, we obtain:

$$
\left(E_{1}^{*}, E_{2}^{*}\right)=\left(E_{1}^{s_{4}}, E_{2}^{s_{4}}\right)=\left(2 E_{\mathrm{av}}, 0\right)
$$

2) $\sigma_{2}^{2}>\sigma_{1}^{2}$ : In this case, it can be verified that $\operatorname{SNR}\left(E_{1}^{s_{3}}, E_{2}^{s_{3}}\right)>\operatorname{SNR}\left(E_{1}^{s_{4}}, E_{2}^{s_{4}}\right)$ and $\operatorname{SNR}\left(E_{1}^{s_{3}}, E_{2}^{s_{3}}\right)>$ $\operatorname{SNR}\left(E_{1}^{s_{2}}, E_{2}^{s_{2}}\right)>\operatorname{SNR}\left(E_{1}^{s_{1}}, E_{2}^{s_{1}}\right)$. So, we obtain:

$$
\left(E_{1}^{*}, E_{2}^{*}\right)=\left(E_{1}^{s_{3}}, E_{2}^{s_{3}}\right)=\left(0,2 E_{\mathrm{av}}\right)
$$

3) $\sigma_{1}^{2}=\sigma_{2}^{2}$ : Finally, if the two transmit-receive wireless links have the same fading parameters, i.e., they are identically distributed but are not necessarily uncorrelated, then we have $\left(E_{1}^{*}, E_{2}^{*}\right)=\left(E_{1}^{s_{3}}, E_{2}^{s_{3}}\right)=\left(E_{1}^{s_{4}}, E_{2}^{s_{4}}\right)$. 


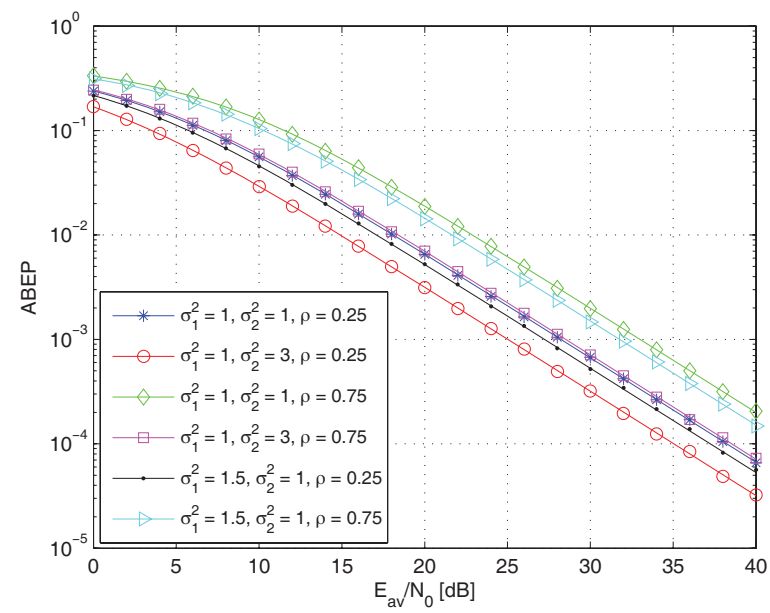

Fig. 1. SSK modulation: ABEP against $E_{\text {av }} / N_{0}$. Solid lines denote the analytical model and markers Monte Carlo simulations.

\section{Performance Gain}

The optimal power allocation solution in Sections III-B1III-B3 illustrates that for a $2 \times 1$ MIMO system the performance can be improved by replacing the conventional uniform power allocation strategy (i.e., $E_{1}=E_{2}=E_{\text {av }}$ ) with an onoff spatial constellation mapping of the information bits to the transmit-antennas. For this reason, the SSK modulation scheme with optimal power allocation introduced in this Letter is denoted by OOSSK modulation.

Let us now compute the asymptotic (i.e., when $\bar{\gamma} E_{\text {av }} \gg$ 1) performance gain achieved by OOSSK modulation with respect to SSK modulation. Let $\mathrm{ABEP}_{\mathrm{SSK}}^{\infty} \cong$ $\left[4 \bar{\gamma}\left(E_{\mathrm{av}} \sigma_{1}^{2}+E_{\mathrm{av}} \sigma_{2}^{2}-2 \rho E_{\mathrm{av}} \sigma_{1} \sigma_{2}\right)\right]^{-1}$ and $\mathrm{ABEP}_{\mathrm{OOSSK}}^{\infty} \cong$ $\left[4 \bar{\gamma}\left(2 E_{\mathrm{av}} \sigma_{M}^{2}\right)\right]^{-1}$ with $\sigma_{M}^{2}=\sigma_{1}^{2}$ if $\sigma_{1}^{2} \geq \sigma_{2}^{2}$ and $\sigma_{M}^{2}=\sigma_{2}^{2}$ if $\sigma_{2}^{2}>\sigma_{1}^{2}$ be the ABEP of SSK modulation and OOSSK modulation for large SNRs, respectively. Then, we have:

$$
\mathrm{SNR}_{\text {gain }} \cong 10 \log _{10}\left[\frac{2 \sigma_{M}^{2}}{\sigma_{1}^{2}+\sigma_{2}^{2}-2 \rho \sigma_{1} \sigma_{2}}\right] \geq 0
$$

\section{NUMERICAL EXAMPLES}

In Fig. 1 and Fig. 2, we show some numerical results to validate the analytical derivation and the optimality of OOSSK modulation with respect to conventional SSK modulation. By comparing both figures, we can readily observe that OOSSK modulation offers better performance than SSK modulation, and the SNR improvement depends on the triple, i.e., the average fading parameters, $\left(\sigma_{1}^{2}, \sigma_{2}^{2}, \rho\right)$. By direct inspection, we note that, for the analyzed system setup, the performance improvement is in the range $\mathrm{SNR}_{\text {gain }} \cong$ $1.25 \mathrm{~dB}$ if $\left(\sigma_{1}^{2}, \sigma_{2}^{2}, \rho\right)=(1,1,0.25)$ and $\mathrm{SNR}_{\text {gain }} \cong 6.55 \mathrm{~dB}$ if $\left(\sigma_{1}^{2}, \sigma_{2}^{2}, \rho\right)=(1.5,1,0.75)$. These performance gains perfectly agree with (11). Also, from (11) we notice that the performance gain (or the energy efficiency) of OOSSK modulation depends on the power imbalance ratio $\sigma_{1}^{2} / \sigma_{2}^{2}$, and improves with increasing values of $\rho$. Furthermore, as expected from (7) and (8), from Fig. 2 we conclude that an additional benefit of OOSSK modulation with respect to SSK modulation is that the performance of the former scheme is independent of the spatial correlation of the channel fading.

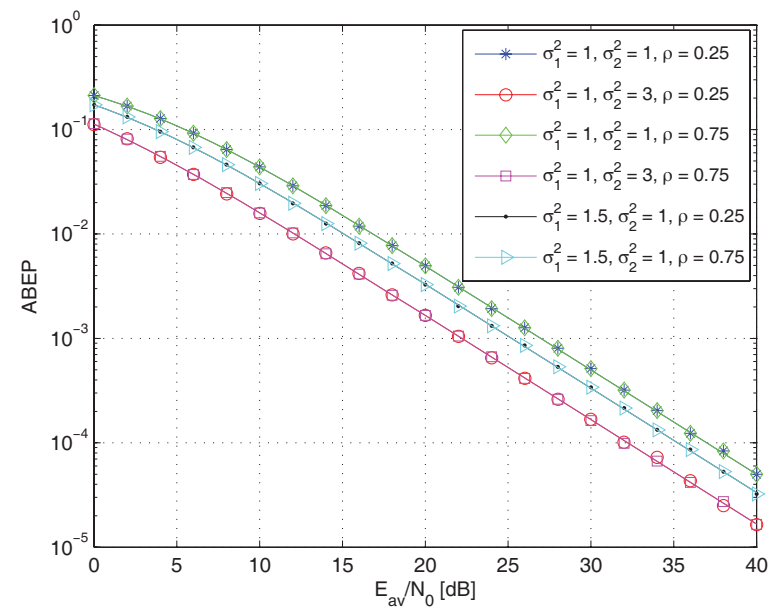

Fig. 2. OOSSK modulation: ABEP against $E_{\mathrm{av}} / N_{0}$. Solid lines denote the analytical model and markers Monte Carlo simulations.

\section{CONCLUSION}

In this Letter, we have shown that the performance of SSK modulation can be improved via suitable opportunistic power allocation methods, which require the transmitter to have some a priori average channel state information knowledge. The optimal power allocation problem has been solved in closed-form for a $2 \times 1$ MIMO scheme, and a significant performance improvement has been observed for the same average power consumed at the transmitter. Moving from the promising results of this Letter, ongoing research is concerned with the analysis of the optimal power allocation problem for arbitrary transmit- and receive-antennas.

\section{ACKNOWLEDGMENT}

We gratefully acknowledge support from the EPSRC (EP/G011788/1) for this work. Harald Haas acknowledges the Scottish Funding Council support of his position within the Edinburgh Research Partnership in Engineering and Mathematics between the University of Edinburgh and Heriot Watt University.

\section{REFERENCES}

[1] Y. Chau and S.-H. Yu, "Space modulation on wireless fading channels," in Proc. IEEE Veh. Technol. Conf. - Fall, vol. 3, pp. 1668-1671, Oct. 2001.

[2] H. Haas, E. Costa, and E. Schultz, "Increasing spectral efficiency by data multiplexing using antennas arrays," in Proc. IEEE Int. Symp. Personal, Indoor, Mobile Radio Commun., vol. 2, pp. 610-613, Sep. 2002.

[3] R. Mesleh, H. Haas, S. Sinanovic, C. Ahn, and S. Yun, "Spatial modulation," IEEE Trans. Veh. Technol., vol. 57, no. 4, pp. 2228-2241, July 2008.

[4] J. Jeganathan, A. Ghrayeb, and L. Szczecinski, "Spatial modulation: optimal detection and performance analysis," IEEE Commun. Lett., vol. 12, no. 8, pp. 545-547, Aug. 2008.

[5] J. Jeganathan, A. Ghrayeb, L. Szczecinski, and A. Ceron, "Space shift keying modulation for MIMO channels," IEEE Trans. Wireless Commun., vol. 8, no. 7, pp. 3692-3703, July 2009

[6] M. Di Renzo and H. Haas, "Performance comparison of different spatial modulation schemes in correlated fading channels," in Proc. IEEE Int. Commun. Conf., Cape Town, South Africa, May 2010.

[7] M. Di Renzo and H. Haas, "A general framework for performance analysis of space shift keying (SSK) modulation for MISO systems over correlated Nakagami- $m$ fading channels," IEEE Trans. Commun., 2010, accepted, to appear.

[8] D. P. Bertsekas, Constrained Optimization and Lagrange Multiplier Methods. Athena Scientific, 1st ed., Jan. 1996. 